\title{
Measurements of the Speed of Sound in Liquid and Supercritical Ethane
}

\author{
A. El Hawary and K. Meier ${ }^{1)}$ \\ Institut für Thermodynamik, Helmut-Schmidt-Universität/Universität der Bundeswehr Hamburg \\ Holstenhofweg 85, 22043 Hamburg, Germany
}

\begin{abstract}
Comprehensive and accurate measurements of the speed of sound in pure ethane have been carried out in the liquid and supercritical regions by a double-path-length pulse-echo technique. The measured data cover the temperature range from $240 \mathrm{~K}$ to $420 \mathrm{~K}$ with pressures up to $100 \mathrm{MPa}$. The expanded measurement uncertainties at the $95 \%$ confidence level amount to $2.1 \mathrm{mK}$ for temperature, $0.007 \%$ for pressure, and $0.01 \%$ for speed of sound with the exception of a few state points in the vicinity of the critical point, where the uncertainty increases up to $0.016 \%$. The quality of the measurements is demonstrated by comparisons with literature data and the fundamental equation of state for ethane by D. Bücker, W. Wagner [J. Phys. Chem. Ref. Data 35 (2006) 205-266].
\end{abstract}

Keywords: ethane, high pressure, pulse-echo method, speed of sound

\section{Introduction}

Ethane is an important fluid with many industrial and scientific applications. For example, it is one of the most important basic substances in many production processes in the chemical industry, and it occurs as the second main component in natural gas beside methane. Moreover, it often serves as a reference fluid for developing equations of state or is used for validating models of intermolecular forces in molecular simulations. Therefore, precise knowledge of its thermodynamic properties is desirable.

Thermodynamic properties of a fluid are best represented by fundamental equations of state, from which all thermodynamic properties can be calculated. For developing and optimizing fundamental equation of state accurate speed-of-sound data sets in the liquid phase are very useful. They contribute to improving the description of the liquid region, the extrapolation behavior and the representation of caloric properties. For ethane, the most accurate fundamental equation of state was published by Bücker and Wagner [1]. For the optimization of the equation of state in the liquid phase, only one comprehensive speed-of-sound data set by Tsumura and Straty 22 was available. These data were measured with a sing-around pulse-echo technique, have an uncertainty of $0.05 \%$ and cover the low temperature range between $100 \mathrm{~K}$ and $323 \mathrm{~K}$ with pressures up to $37 \mathrm{MPa}$. Besides, Trusler and Costa Gomez [3] measured a few data with a spherical resonator on the supercritical isotherm $350 \mathrm{~K}$ up to $20 \mathrm{MPa}$. At low pressures in the liquid phase, the Bücker and Wagner equation of state describes the speed of sound with an uncertainty of $0.15 \%$. At pressures above $40 \mathrm{MPa}$ the uncertainties increase

1) Corresponding author Email address: karsten.meier@hsu-hh.de 
to $1 \%$ up to $100 \mathrm{MPa}$, and even reach $5 \%$ at higher pressures. With our speed-of-sound instrument [4, 5] very accurate speed-of-sound data with uncertainties well below $0.05 \%$ were already measured for several pure fluids, e.g. [6, 7]. Thus, the aim of this work was to extend the measurement range for the speed of sound in liquid and supercritical ethane to higher pressures up to $100 \mathrm{MPa}$ and higher temperatures up to $420 \mathrm{~K}$. The new data data can subsequently be used to improve the formulation of the Helmholtz energy for ethane.

\section{Experimental Procedure}

Our speed-of-sound instrument, the calibration procedure, and the analysis of measurements have been described in detail in refs. 4, 5. The measurement principle of our speed-of-sound sensor is based on a double-path-length pulse-echo technique, which was first introduced by Muringer et al. [8]. A piezoelectric quartz crystal with a resonance frequency of $8 \mathrm{MHz}$ is employed as a sound emitter and receiver. In the analysis of the speed-of-sound measurements, corrections for changes of the distances in the sensor with temperature, for compression of the sensor with pressure, and for diffraction effects are applied as described in ref. [4].

The speed-of-sound sensor is housed in a pressure vessel, which is thermostated in a circulating liquid-bath thermostat. The temperature inside the pressure vessel is kept constant within $0.5 \mathrm{mK}$ during the course of one measurement. The temperature was measured by a 25-Ohm standard platinum resistance thermometer in the wall of the pressure vessel. The thermometer was calibrated on the ITS90 , and the uncertainty of the temperature measurement is estimated to be $2.1 \mathrm{mK}$. The resistance of the thermometer was measured with an ASL-F18 bridge system with calibrated reference resistors. The pressure inside the pressure vessel was measured with a nitrogen-operated gas pressure balance. The pressure balance was coupled to the sample liquid via a differential pressure null indicator (Ruska membrane type cell). The uncertainty of the pressure measurement is less than $70 \mathrm{ppm}$. Both temperature and pressure measurement uncertainties are for a $95 \%$ confidence level.

The acoustic path length $\Delta l=2\left(l_{2}-l_{1}\right)$ at zero pressure and $273.15 \mathrm{~K}$ and the temperature depen-

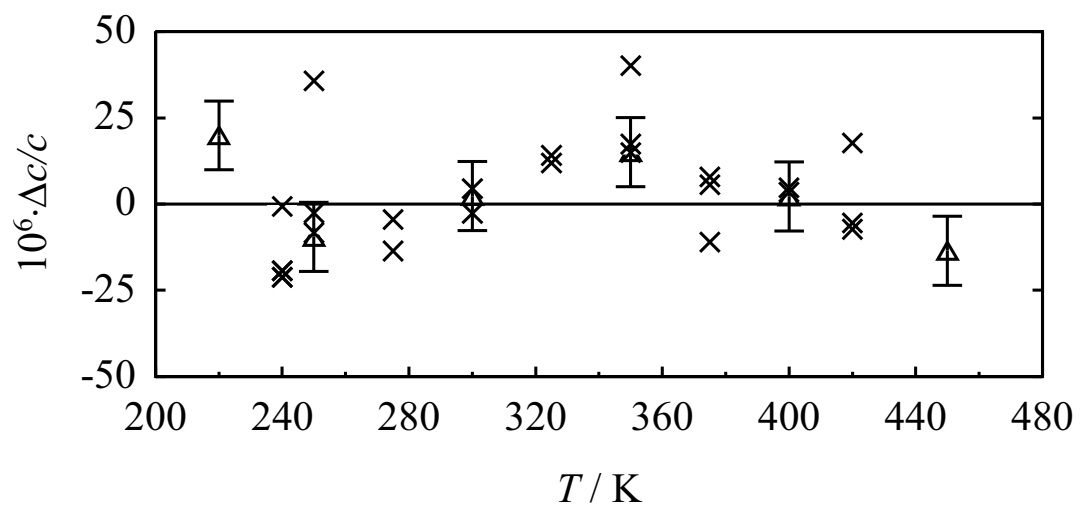

Fig. 1. Relative deviations of measured speeds of sound in gaseous argon from the fundamental equation of state of Tegeler et al. after adjustment of the acoustic path length. Legend: $X$, This work; $\triangle$, EstradaAlexanders and Trusler. 
dent thermal expansion coefficient of the sensor material were determined by calibration measurements in gaseous argon at pressures between $10 \mathrm{MPa}$ and $13 \mathrm{MPa}$ and temperatures between $240 \mathrm{~K}$ and $420 \mathrm{~K}$. Argon was used as a calibration fluid instead of water because it enables to calibrate the sensor in a wider temperature range and to achieve a lower uncertainty of the calibration. With the speedof-sound data of Estrada-Alexanders and Trusler [9, which have a relative uncertainty of $10 \mathrm{ppm}$, accurate references for the speed of sound in argon are available. The analysis of the calibration measurements was started with a calibration function from a previous measurement campaign. Minimization of the deviations of our measurements from the reference data of Estrada-Alexanders and Trusler was achieved by reducing the value of the acoustic path length at zero pressure and $273.15 \mathrm{~K}$ by about $0.014 \%$. The difference to the previous calibration is probably due to the fact that the sensor was disassembled and reassembled before the calibration measurements. After the calibration two complete measurement campaigns with different ethane samples were carried out. These measurements had to be discarded because the samples were contaminated with air. Then the present ethane measurements were conducted, followed by a second calibration with argon which was also carried out and analyzed as described above. It was found that the acoustic path length had increased by $0.035 \%$ since the first calibration. It is assumed that this difference is due to small movements of the crystal in the speed-of-sound sensor during temperature and pressure cycles. To check the reproducibility of the speed of sound after the second calibration, a few measurements at $T=340 \mathrm{~K}$ were repeated since this isotherm was measured first. These data deviated from the speeds of sound of the main measurement campaign by up to 55 ppm when evaluated with the same calibration function for the acoustic path length. This result provided high confidence that the speed-of-sound measurements for ethane are very consistent and are not affected by the rather large difference between the two calibrations. For this reason the ethane measurements were evaluated with the second calibration, and an additional allowance of 55 ppm was added as a systematic contribution to the combined uncertainty of the speed of sound. The observed difference of 55 ppm is probably also due to movements of the crystal during frequent temperature changes in the second calibration.

Fig. 1 shows the deviations of our second series of calibration measurements and of the data of Estrada-Alexanders and Trusler from the accurate fundamental equation of state of Tegeler et al. for argon as a function of temperature after adjustment of the acoustic path length. Our measurements agree with the data of Estrada-Alexanders and Trusler within 20 ppm with the exception of two points, which deviate by $35 \mathrm{ppm}$ and $45 \mathrm{ppm}$ from the data of Estrada-Alexanders and Trusler. These two points were discarded in the analysis of the calibration measurements. Their deviations are covered by the additional allowance of 55 ppm to the uncertainty of the speed of sound described above. The expanded uncertainty (confidence level $95 \%$ ) of the determination of the acoustic path length is estimated to be $40 \mathrm{ppm}$. It includes contributions from the time, temperature and pressure measurements, the uncertainty of the reference data, the deviation of the calibration measurements from the reference data, and the correction of the acoustic path length to zero pressure. Estrada-Alexanders and Trusler did not state whether the reported uncertainty of their speed of sound data is at the $68 \%$ or $95 \%$ confidence level. In our uncertainty analysis, it was considered as an expanded uncertainty at the $95 \%$ confidence level. A detailed summary of all contributions to the measurement uncertainties is given in Table 1 .

For the measurements in ethane, the resolution of the time measurements is less than $2 \mathrm{ppm}$, while 
Table 1. Summary of Uncertainty Budgets.

\begin{tabular}{|c|c|c|}
\hline Source of uncertainty & Value (confidence level=95\%) & Influence \\
\hline \multicolumn{3}{|l|}{ Temperature measurement: } \\
\hline SPRT calibration & $2.0 \mathrm{mK}$ & random \\
\hline Calibration of reference resistance & $0.1 \mathrm{mK}$ & random \\
\hline ASL F18 bridge & $0.1 \mathrm{mK}$ & random \\
\hline Temperature variation in pressure vessel & $0.5 \mathrm{mK}$ & random \\
\hline Total uncertainty & $2.1 \mathrm{mK}$ & \\
\hline \multicolumn{3}{|l|}{ Pressure measurement: } \\
\hline Calibration of pressure balance & $10^{-4} \mathrm{MPa}+4.3 \cdot 10^{-5} \cdot p+2.8 \cdot 10^{-7}$ & $\begin{array}{l}7 \cdot p^{2} / \mathrm{MPa} \\
\text { random }\end{array}$ \\
\hline Differential pressure indicator & $5 \cdot 10^{-6} \cdot p$ & random \\
\hline Hydrostatic pressure correction & $2 \cdot 10^{-6} \cdot p$ & random \\
\hline Ambient pressure measurement & $20 \mathrm{~Pa}$ & random \\
\hline Total uncertainty & $<70 \cdot 10^{-6} \cdot p$ & \\
\hline \multicolumn{3}{|c|}{ Determination of acoustic path length with measurements in argon: } \\
\hline Time difference & $2 \cdot 10^{-6} \cdot \Delta L$ & random \\
\hline Temperature measurement & $6 \cdot 10^{-6} \cdot \Delta L$ & random \\
\hline Pressure measurement & $6 \cdot 10^{-6} \cdot \Delta L$ & random \\
\hline Correction of $\Delta L$ to ambient pressure & $3 \cdot 10^{-6} \cdot \Delta L$ & systematic \\
\hline Diffraction correction & $2 \cdot 10^{-6} \cdot \Delta L$ & systematic \\
\hline Uncertainty of reference data & $10 \cdot 10^{-6} \cdot \Delta L$ & random \\
\hline \multicolumn{3}{|l|}{ Deviation of calibration measurements from } \\
\hline reference data & $20 \cdot 10^{-6} \cdot \Delta L$ & systematic \\
\hline Total uncertainty & $40 \cdot 10^{-6} \cdot \Delta L$ & \\
\hline \multicolumn{3}{|l|}{ Speed-of-sound measurement: } \\
\hline Acoustic path length & $40 \cdot 10^{-6} \cdot c$ & random \\
\hline Pressure dependence of acoustic path length & $\left(25 \cdot 10^{-8} \cdot p / \mathrm{MPa}\right) \cdot c$ & systematic \\
\hline Time difference & $2 \cdot 10^{-6} \cdot c$ & random \\
\hline Diffraction correction & $3 \cdot 10^{-6} \cdot c$ & random \\
\hline Total uncertainty & $\left(45 \cdot 10^{-6}+25 \cdot 10^{-8} \cdot p / \mathrm{MPa}\right) \cdot c$ & \\
\hline
\end{tabular}

the reproducibility of the speed of sound after temperature and pressure cycles during the measurement campaign is less than $25 \mathrm{ppm}$, at high pressures it is even less than $10 \mathrm{ppm}$. It is assumed that the reproducibility of the time measurements is limited by the reproducibility with which the temperature and pressure of a state point can be set in the pressure vessel. The uncertainty of the speed-of-sound measurement is $45 \mathrm{ppm}$ at the $95 \%$ confidence level, excluding contributions from sample impurities, from temperature and pressure measurement uncertainties and the reproducibility after the second calibration.

The ethane sample was purchased from Westfalen, Germany, had a manufacturer specified volume purity better than $99.999 \%$ and was used as delivered. According to the manufacturer's specification the volume purity is as follows: other hydrocarbons $\left(\mathrm{CH}_{4}, \mathrm{C}_{2} \mathrm{H}_{4}, \mathrm{C}_{2} \mathrm{H}_{2}, \mathrm{C}_{3} \mathrm{H}_{8}\right.$, and $\left.\mathrm{C}_{4^{+}}\right): \leq 6 \mathrm{ppm}$, 
nitrogen: $\leq 3$ ppm, carbon dioxide: $\leq 2$ ppm, oxygen: $\leq 1 \mathrm{ppm}$, and water: $\leq 1 \mathrm{ppm}$. To account for sample impurities, an additional allowance of $10 \mathrm{ppm}$ was added to the combined uncertainty of the speed of sound.

\section{Results}

Our measurements cover the liquid and supercritical region between $T=240 \mathrm{~K}$ and $T=420 \mathrm{~K}$ with pressures up to $100 \mathrm{MPa}$ and were conducted along 10 isotherms in steps of $20 \mathrm{~K}$. Additionally, measurements on the critical isotherm at $T_{\text {crit }}=305.32 \mathrm{~K}$ were carried out. In order to compare our measurements with the spherical resonator data of Trusler and Costa Gomez, some data were measured between $13 \mathrm{MPa}$ and $25 \mathrm{MPa}$ at $T=350 \mathrm{~K}$. On subcritical isotherms the lowest pressure was chosen slightly above the vapor pressure of ethane at ambient temperature of about $3.75 \mathrm{MPa}$, while on supercritical isotherms measurements were started at the lowest pressure where a clear signal cancellation could be observed on the oscilloscope. The measurement results are listed in Table 2. Our speed-of-sound data on the eleven completely measured isotherms are shown in Fig. 2 as a function of pressure. In the region of our measurements, the speed of sound ranges from $350 \mathrm{~m} \cdot \mathrm{s}^{-1}$ to $1600 \mathrm{~m} \cdot \mathrm{s}^{-1}$.

Table 2. Results for the speed of sound in ethane.

\begin{tabular}{|c|c|c|c|c|c|c|c|}
\hline$T / \mathrm{K}$ & $p / \mathrm{MPa}$ & $c / \mathrm{m} \cdot \mathrm{s}^{-1}$ & $U_{\mathrm{c}}(c) / \mathrm{m} \cdot \mathrm{s}^{-1}$ & $T / \mathrm{K}$ & $p / \mathrm{MPa}$ & $c / \mathrm{m} \cdot \mathrm{s}^{-1}$ & $U_{\mathrm{c}}(c) / \mathrm{m} \cdot \mathrm{s}^{-1}$ \\
\hline \multicolumn{8}{|c|}{$T=240 \mathrm{~K}$} \\
\hline 240.0012 & 4.10593 & 926.064 & 0.084 & 239.9997 & 25.1199 & 1166.32 & 0.10 \\
\hline 239.9981 & 5.10652 & 941.251 & 0.085 & 240.0001 & 30.1231 & 1209.20 & 0.11 \\
\hline 239.9980 & 6.10717 & 955.831 & 0.086 & 240.0001 & 35.1264 & 1248.93 & 0.11 \\
\hline 239.9978 & 7.10781 & 969.878 & 0.087 & 240.0001 & 40.1303 & 1286.06 & 0.12 \\
\hline 239.9978 & 8.10848 & 983.446 & 0.088 & 240.0003 & 45.1335 & 1320.97 & 0.12 \\
\hline 239.9977 & 9.10914 & 996.573 & 0.089 & 240.0008 & 50.1368 & 1353.97 & 0.12 \\
\hline 239.9985 & 10.1100 & 1009.31 & 0.09 & 240.0010 & 60.1434 & 1415.23 & 0.13 \\
\hline 240.0013 & 10.1098 & 1009.29 & 0.09 & 240.0011 & 70.1499 & 1471.28 & 0.14 \\
\hline 239.9988 & 12.6117 & 1039.51 & 0.09 & 240.0000 & 80.1574 & 1523.13 & 0.14 \\
\hline 239.9990 & 15.1133 & 1067.76 & 0.09 & 240.0000 & 90.1641 & 1571.51 & 0.15 \\
\hline 239.9994 & 17.6150 & 1094.36 & 0.10 & 240.0003 & 100.171 & 1616.94 & 0.16 \\
\hline 239.9993 & 20.1166 & 1119.53 & 0.10 & & & & \\
\hline \multicolumn{8}{|c|}{$T=260 \mathrm{~K}$} \\
\hline 259.9961 & 4.10520 & 758.654 & 0.073 & 259.9974 & 25.1182 & 1055.06 & 0.10 \\
\hline 259.9963 & 4.10521 & 758.657 & 0.073 & 259.9973 & 30.1216 & 1103.48 & 0.10 \\
\hline 259.9964 & 5.10587 & 779.780 & 0.074 & 259.9972 & 35.1250 & 1147.75 & 0.10 \\
\hline 259.9964 & 6.10658 & 799.566 & 0.075 & 259.9969 & 40.1292 & 1188.69 & 0.11 \\
\hline 259.9962 & 7.10727 & 818.209 & 0.076 & 259.9969 & 45.1326 & 1226.87 & 0.11 \\
\hline
\end{tabular}


Table 2. (continued)

\begin{tabular}{|c|c|c|c|c|c|c|c|}
\hline$T / \mathrm{K}$ & $p / \mathrm{MPa}$ & $c / \mathrm{m} \cdot \mathrm{s}^{-1}$ & $U_{\mathrm{c}}(c) / \mathrm{m} \cdot \mathrm{s}^{-1}$ & $T / \mathrm{K}$ & $p / \mathrm{MPa}$ & $c / \mathrm{m} \cdot \mathrm{s}^{-1}$ & $U_{\mathrm{c}}(c) / \mathrm{m} \cdot \mathrm{s}^{-1}$ \\
\hline 259.9963 & 8.10795 & 835.879 & 0.078 & 259.9971 & 50.1361 & 1262.73 & 0.12 \\
\hline 259.9959 & 9.10859 & 852.699 & 0.079 & 259.9969 & 60.1429 & 1328.74 & 0.12 \\
\hline 259.9985 & 10.1088 & 868.730 & 0.080 & 259.9992 & 60.1429 & 1328.72 & 0.12 \\
\hline 259.9959 & 10.1092 & 868.763 & 0.080 & 259.9994 & 60.1429 & 1328.72 & 0.12 \\
\hline 259.9970 & 12.6096 & 906.138 & 0.083 & 259.9969 & 70.1498 & 1388.63 & 0.13 \\
\hline 259.9971 & 15.1113 & 940.293 & 0.085 & 259.9969 & 80.1567 & 1443.69 & 0.14 \\
\hline 259.9974 & 17.6130 & 971.860 & 0.088 & 259.9968 & 90.1636 & 1494.80 & 0.15 \\
\hline 259.9971 & 20.1147 & 1001.30 & 0.09 & 259.9996 & 100.170 & 1542.59 & 0.15 \\
\hline \multicolumn{8}{|c|}{$T=280 \mathrm{~K}$} \\
\hline 280.0052 & 4.10515 & 566.797 & 0.064 & 280.0055 & 25.1195 & 949.349 & 0.088 \\
\hline 280.0052 & 5.10580 & 601.255 & 0.064 & 280.0058 & 30.1229 & 1003.67 & 0.09 \\
\hline 280.0053 & 6.10650 & 631.325 & 0.065 & 280.0057 & 35.1263 & 1052.61 & 0.10 \\
\hline 280.0055 & 7.10721 & 658.247 & 0.066 & 280.0061 & 40.1297 & 1097.38 & 0.10 \\
\hline 280.0054 & 8.10789 & 682.772 & 0.068 & 280.0058 & 45.1329 & 1138.77 & 0.11 \\
\hline 280.0049 & 9.10855 & 705.402 & 0.069 & 280.0059 & 50.1362 & 1177.40 & 0.11 \\
\hline 280.0054 & 10.1092 & 726.480 & 0.070 & 280.0057 & 60.1429 & 1247.95 & 0.12 \\
\hline 280.0051 & 12.6109 & 773.922 & 0.073 & 279.9958 & 70.1496 & 1311.50 & 0.13 \\
\hline 280.0057 & 15.1126 & 815.712 & 0.077 & 279.9965 & 80.1563 & 1369.52 & 0.13 \\
\hline 280.0058 & 17.6144 & 853.356 & 0.080 & 279.9967 & 90.1630 & 1423.14 & 0.14 \\
\hline 280.0057 & 20.1161 & 887.783 & 0.082 & 279.9968 & 100.169 & 1473.10 & 0.15 \\
\hline \multicolumn{8}{|c|}{$T=300 \mathrm{~K}$} \\
\hline 299.9958 & 5.10486 & 368.914 & 0.067 & 299.9981 & 30.1220 & 910.875 & 0.086 \\
\hline 299.9955 & 6.10563 & 434.503 & 0.060 & 299.9985 & 35.1255 & 964.446 & 0.091 \\
\hline 299.9953 & 7.10643 & 481.738 & 0.059 & 299.9981 & 40.1289 & 1012.92 & 0.10 \\
\hline 299.9955 & 8.10714 & 520.103 & 0.060 & 299.9983 & 45.1326 & 1057.39 & 0.10 \\
\hline 299.9960 & 9.10782 & 553.015 & 0.061 & 299.9986 & 50.1359 & 1098.61 & 0.11 \\
\hline 299.9959 & 10.1085 & 582.153 & 0.062 & 299.9986 & 60.1428 & 1173.35 & 0.11 \\
\hline 299.9960 & 12.6102 & 644.019 & 0.065 & 299.9984 & 70.1495 & 1240.16 & 0.12 \\
\hline 299.9959 & 15.1119 & 695.622 & 0.069 & 299.9992 & 80.1563 & 1300.86 & 0.13 \\
\hline 299.9963 & 17.6133 & 740.509 & 0.072 & 299.9992 & 90.1631 & 1356.74 & 0.14 \\
\hline 299.9981 & 20.1151 & 780.577 & 0.075 & 299.9993 & 100.170 & 1408.64 & 0.14 \\
\hline 299.9981 & 25.1186 & 850.525 & 0.081 & & & & \\
\hline \multicolumn{8}{|c|}{$T=305.32 \mathrm{~K}$} \\
\hline 305.3173 & 6.10496 & 370.857 & 0.062 & 305.3188 & 30.1222 & 887.499 & 0.085 \\
\hline 305.3176 & 7.10578 & 429.831 & 0.059 & 305.3189 & 35.1254 & 942.256 & 0.090 \\
\hline 305.3174 & 8.10655 & 474.477 & 0.058 & 305.3189 & 40.129 & 991.671 & 0.095 \\
\hline
\end{tabular}


Table 2. (continued)

\begin{tabular}{|c|c|c|c|c|c|c|c|}
\hline$T / \mathrm{K}$ & $p / \mathrm{MPa}$ & $c / \mathrm{m} \cdot \mathrm{s}^{-1}$ & $U_{\mathrm{c}}(c) / \mathrm{m} \cdot \mathrm{s}^{-1}$ & $T / \mathrm{K}$ & $p / \mathrm{MPa}$ & $c / \mathrm{m} \cdot \mathrm{s}^{-1}$ & $U_{\mathrm{c}}(c) / \mathrm{m} \cdot \mathrm{s}^{-1}$ \\
\hline 305.3172 & 9.10732 & 511.481 & 0.059 & 305.3192 & 45.1323 & 1036.91 & 0.10 \\
\hline 305.3175 & 10.108 & 543.524 & 0.060 & 305.3196 & 50.1357 & 1078.77 & 0.10 \\
\hline 305.3176 & 12.6098 & 610.162 & 0.063 & 305.3194 & 60.1424 & 1154.56 & 0.11 \\
\hline 305.3175 & 15.1116 & 664.739 & 0.067 & 305.3197 & 70.1489 & 1222.17 & 0.12 \\
\hline 305.3175 & 17.6134 & 711.717 & 0.070 & 305.3200 & 80.1554 & 1283.54 & 0.13 \\
\hline 305.3175 & 20.1152 & 753.370 & 0.073 & 305.3202 & 90.1618 & 1339.96 & 0.14 \\
\hline 305.3173 & 25.1187 & 825.573 & 0.079 & 305.3202 & 100.168 & 1392.33 & 0.14 \\
\hline 305.3185 & 25.1190 & 825.573 & 0.079 & & & & \\
\hline \multicolumn{8}{|c|}{$T=320 \mathrm{~K}$} \\
\hline 320.0042 & 8.10853 & 346.325 & 0.055 & 320.0047 & 20.1171 & 681.812 & 0.069 \\
\hline 320.0043 & 9.10928 & 397.386 & 0.055 & 320.0049 & 25.1205 & 760.047 & 0.075 \\
\hline 319.9967 & 9.10820 & 397.392 & 0.055 & 320.0048 & 30.1239 & 826.137 & 0.081 \\
\hline 319.9969 & 9.10822 & 397.386 & 0.055 & 320.0050 & 35.1273 & 884.000 & 0.086 \\
\hline 320.0044 & 10.1100 & 438.915 & 0.056 & 320.0049 & 40.1308 & 935.854 & 0.091 \\
\hline 320.0044 & 11.1107 & 474.377 & 0.057 & 320.0046 & 45.1341 & 983.085 & 0.096 \\
\hline 320.0044 & 12.1114 & 505.620 & 0.058 & 320.0048 & 50.1375 & 1026.62 & 0.10 \\
\hline 319.9971 & 12.1104 & 505.627 & 0.058 & 320.0047 & 60.1443 & 1105.08 & 0.11 \\
\hline 320.0047 & 13.1121 & 533.741 & 0.059 & 320.0047 & 70.1514 & 1174.77 & 0.12 \\
\hline 320.0045 & 14.1128 & 559.399 & 0.061 & 320.0048 & 80.1582 & 1237.82 & 0.12 \\
\hline 320.0043 & 15.1135 & 583.107 & 0.062 & 320.0049 & 90.1650 & 1295.66 & 0.13 \\
\hline 319.9970 & 15.1124 & 583.116 & 0.062 & 320.0051 & 100.172 & 1349.23 & 0.14 \\
\hline 320.0044 & 17.6152 & 635.895 & 0.066 & & & & \\
\hline \multicolumn{8}{|c|}{$T=340 \mathrm{~K}$} \\
\hline 340.0030 & 10.1090 & 324.097 & 0.045 & 340.0044 & 25.1188 & 679.706 & 0.070 \\
\hline 340.0038 & 10.1090 & 324.083 & 0.045 & 340.0048 & 30.1222 & 750.561 & 0.076 \\
\hline 340.0029 & 11.1097 & 362.179 & 0.049 & 340.0044 & 35.1256 & 812.002 & 0.081 \\
\hline 340.0026 & 12.1104 & 397.205 & 0.051 & 340.0043 & 40.1289 & 866.676 & 0.086 \\
\hline 340.0027 & 13.1110 & 429.062 & 0.053 & 340.0045 & 45.1322 & 916.206 & 0.091 \\
\hline 340.0036 & 14.1114 & 458.141 & 0.054 & 340.0043 & 50.1369 & 961.680 & 0.096 \\
\hline 340.0034 & 15.1120 & 484.909 & 0.056 & 340.0041 & 60.1439 & 1043.25 & 0.10 \\
\hline 340.0040 & 15.1125 & 484.913 & 0.056 & 340.0043 & 70.1508 & 1115.34 & 0.11 \\
\hline 340.0039 & 17.6137 & 543.915 & 0.060 & 340.0043 & 80.1578 & 1180.36 & 0.12 \\
\hline 340.0042 & 17.6142 & 543.923 & 0.060 & 340.0041 & 90.1647 & 1239.84 & 0.13 \\
\hline 340.0040 & 20.1154 & 594.616 & 0.063 & 340.0040 & 100.172 & 1294.82 & 0.14 \\
\hline \multicolumn{8}{|c|}{$T=350 \mathrm{~K}$} \\
\hline 350.0020 & 13.1114 & 388.893 & 0.049 & 350.0024 & 17.1142 & 493.336 & 0.056 \\
\hline
\end{tabular}


Table 2. (continued)

\begin{tabular}{|c|c|c|c|c|c|c|c|}
\hline$T / \mathrm{K}$ & $p / \mathrm{MPa}$ & $c / \mathrm{m} \cdot \mathrm{s}^{-1}$ & $U_{\mathrm{c}}(c) / \mathrm{m} \cdot \mathrm{s}^{-1}$ & $T / \mathrm{K}$ & $p / \mathrm{MPa}$ & $c / \mathrm{m} \cdot \mathrm{s}^{-1}$ & $U_{\mathrm{c}}(c) / \mathrm{m} \cdot \mathrm{s}^{-1}$ \\
\hline 350.0020 & 14.1120 & 417.627 & 0.051 & 350.0023 & 18.1149 & 515.598 & 0.058 \\
\hline 350.0033 & 15.1124 & 444.518 & 0.053 & 350.0021 & 19.1156 & 536.645 & 0.059 \\
\hline 350.0027 & 15.1124 & 444.518 & 0.053 & 350.0023 & 20.1162 & 556.623 & 0.061 \\
\hline 350.0020 & 15.1127 & 444.535 & 0.053 & 350.0019 & 22.6180 & 602.591 & 0.064 \\
\hline 350.0023 & 16.1135 & 469.707 & 0.055 & 350.0025 & 25.1197 & 643.969 & 0.067 \\
\hline \multicolumn{8}{|c|}{$T=360 \mathrm{~K}$} \\
\hline 360.0045 & 11.1091 & 308.749 & 0.035 & 359.9966 & 30.1216 & 685.231 & 0.071 \\
\hline 360.0049 & 11.1089 & 308.751 & 0.035 & 359.9960 & 35.1260 & 749.097 & 0.077 \\
\hline 360.0048 & 12.1098 & 333.419 & 0.040 & 359.9959 & 40.1295 & 805.780 & 0.082 \\
\hline 360.0053 & 13.1104 & 359.771 & 0.044 & 359.9959 & 45.1330 & 856.995 & 0.087 \\
\hline 360.0049 & 14.1111 & 386.181 & 0.047 & 359.9959 & 50.1364 & 903.907 & 0.092 \\
\hline 360.0053 & 15.1118 & 411.730 & 0.049 & 359.9957 & 60.1432 & 987.833 & 0.101 \\
\hline 360.0058 & 17.6135 & 470.763 & 0.054 & 359.9959 & 70.1501 & 1061.82 & 0.11 \\
\hline 360.0059 & 20.1151 & 522.916 & 0.058 & 359.9959 & 80.1569 & 1128.38 & 0.12 \\
\hline 359.9961 & 20.1155 & 522.955 & 0.058 & 359.9960 & 90.1635 & 1189.16 & 0.12 \\
\hline 359.9966 & 22.6167 & 569.439 & 0.062 & 359.9963 & 100.170 & 1245.27 & 0.13 \\
\hline 359.9967 & 25.1184 & 611.431 & 0.065 & & & & \\
\hline \multicolumn{8}{|c|}{$T=380 \mathrm{~K}$} \\
\hline 379.9982 & 15.1122 & 370.700 & 0.042 & 379.9964 & 35.1260 & 695.570 & 0.073 \\
\hline 379.9983 & 16.1129 & 390.923 & 0.045 & 379.9962 & 40.1295 & 753.227 & 0.078 \\
\hline 379.9982 & 17.1137 & 411.213 & 0.047 & 379.9962 & 45.1329 & 805.392 & 0.084 \\
\hline 379.9984 & 18.1144 & 431.245 & 0.049 & 379.9966 & 50.1363 & 853.187 & 0.088 \\
\hline 379.9984 & 19.1152 & 450.840 & 0.051 & 379.9968 & 60.1431 & 938.665 & 0.097 \\
\hline 379.9972 & 20.1158 & 469.906 & 0.053 & 379.9965 & 70.1498 & 1013.96 & 0.11 \\
\hline 379.9970 & 22.6176 & 515.025 & 0.057 & 379.9993 & 80.1574 & 1081.64 & 0.11 \\
\hline 379.9971 & 25.1193 & 556.627 & 0.061 & 379.9993 & 90.1643 & 1143.40 & 0.12 \\
\hline 379.9968 & 27.6209 & 595.084 & 0.064 & 379.9990 & 100.171 & 1200.37 & 0.13 \\
\hline 379.9968 & 30.1226 & 630.815 & 0.067 & & & & \\
\hline \multicolumn{8}{|c|}{$T=400 \mathrm{~K}$} \\
\hline 400.0010 & 15.1122 & 355.737 & 0.036 & 399.9986 & 35.1258 & 651.397 & 0.070 \\
\hline 400.0009 & 16.1130 & 370.546 & 0.039 & 399.9988 & 40.1288 & 708.909 & 0.075 \\
\hline 399.9986 & 17.1138 & 386.281 & 0.042 & 399.9992 & 45.1323 & 761.231 & 0.080 \\
\hline 399.9990 & 18.1144 & 402.564 & 0.044 & 399.9993 & 50.1356 & 809.310 & 0.085 \\
\hline 399.9985 & 19.1151 & 419.112 & 0.046 & 399.9990 & 60.1425 & 895.499 & 0.094 \\
\hline 399.9988 & 20.1157 & 435.709 & 0.048 & 399.9992 & 70.1493 & 971.531 & 0.103 \\
\hline 399.9987 & 22.6174 & 476.551 & 0.053 & 399.9998 & 80.1560 & 1039.913 & 0.11 \\
\hline
\end{tabular}


Table 2. (continued)

\begin{tabular}{cccccccc}
\hline$T / \mathrm{K}$ & $p / \mathrm{MPa}$ & $c / \mathrm{m} \cdot \mathrm{s}^{-1}$ & $U_{\mathrm{c}}(c) / \mathrm{m} \cdot \mathrm{s}^{-1}$ & $T / \mathrm{K}$ & $p / \mathrm{MPa}$ & $c / \mathrm{m} \cdot \mathrm{s}^{-1}$ & $U_{\mathrm{c}}(c) / \mathrm{m} \cdot \mathrm{s}^{-1}$ \\
\hline 399.9986 & 25.1191 & 515.631 & 0.057 & 399.9998 & 90.1626 & 1102.310 & 0.12 \\
399.9987 & 27.6208 & 552.587 & 0.060 & 400.0003 & 100.169 & 1159.871 & 0.13 \\
399.9984 & 30.1224 & 587.434 & 0.064 & & & & \\
& & & & & & & \\
420.0020 & 15.1106 & 355.162 & 0.034 & 420.0030 & 35.1249 & 615.947 & 0.066 \\
420.0021 & 16.1113 & 365.770 & 0.036 & 420.0036 & 40.1284 & 672.253 & 0.072 \\
420.0023 & 17.1125 & 377.505 & 0.038 & 420.0033 & 45.1318 & 723.939 & 0.077 \\
420.0025 & 18.1132 & 390.104 & 0.040 & 420.0034 & 50.1352 & 771.705 & 0.082 \\
420.0027 & 19.1139 & 403.343 & 0.042 & 420.0034 & 60.1421 & 857.752 & 0.091 \\
420.0027 & 20.1146 & 417.021 & 0.044 & 420.0032 & 70.1495 & 933.942 & 0.100 \\
420.0028 & 22.6163 & 452.115 & 0.049 & 420.0036 & 80.1565 & 1002.60 & 0.11 \\
420.0027 & 25.1181 & 487.213 & 0.053 & 420.0035 & 90.1635 & 1065.31 & 0.12 \\
420.0029 & 27.6198 & 521.412 & 0.057 & 420.0035 & 100.170 & 1123.20 & 0.12 \\
420.0030 & 30.1215 & 554.325 & 0.060 & & & & \\
\hline
\end{tabular}

${ }^{\text {a }}$ Uncertainty of temperature: $u(T)=2.1 \mathrm{mK}$; relative uncertainty of pressure: $u_{\mathrm{r}}(p)=0.008 \%$ (all uncertainties refer to a level of confidence $=95 \%$ ).

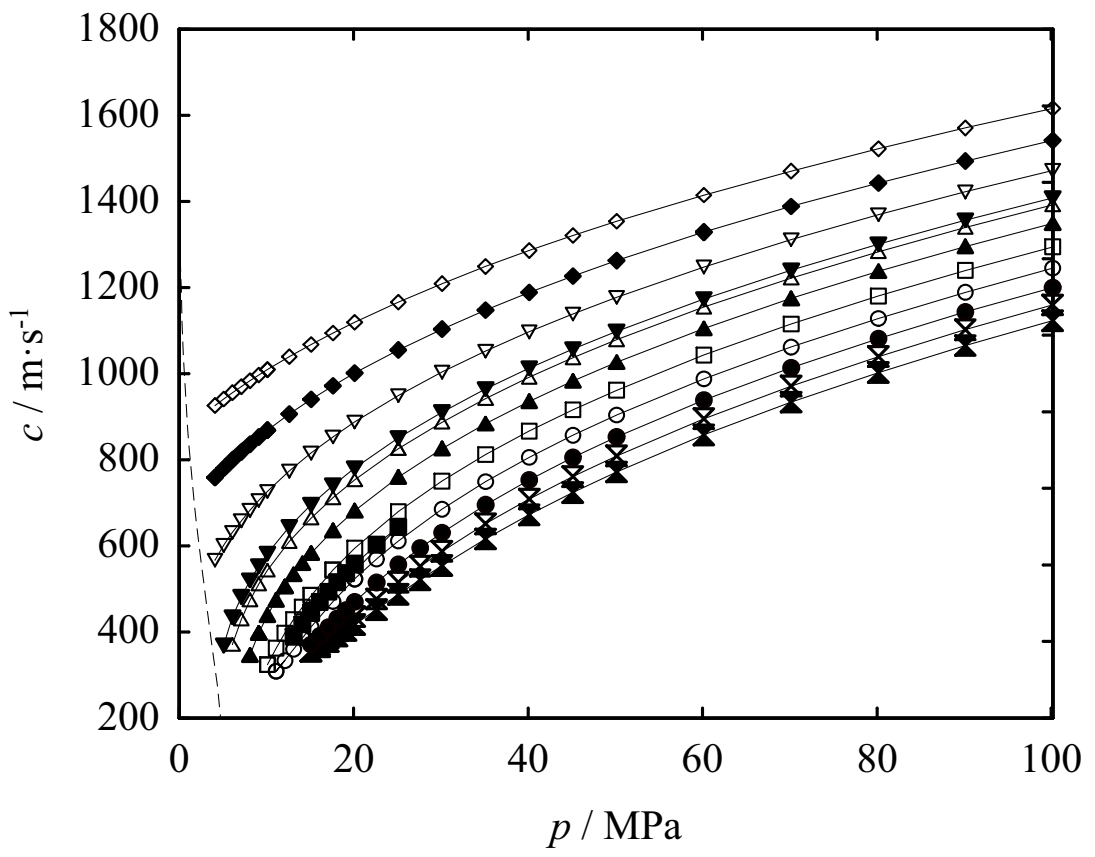

Fig. 2. The speed of sound in ethane as a function of pressure for all measured isotherms. Legend: $\diamond, 240 \mathrm{~K}$;

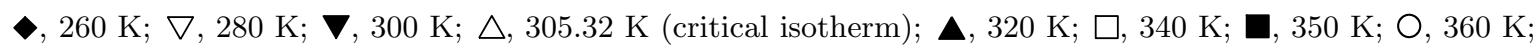

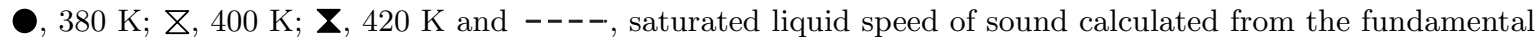
equation of state of Bücker and Wagner. 
For each measured state point a detailed analysis of the combined uncertainty of the speed-of-sound measurement was carried out. The uncertainty contributions to the speed of sound due to the uncertainties of temperature and pressure measurements were estimated by the equation of state of Bücker and Wagner [1]. The combined uncertainty is generally less than $0.01 \%$, including the uncertainties of the temperature and pressure measurement and the influences due to sample impurities. Only at low pressures in the critical region it is larger, increasing up to $0.016 \%$. These uncertainty estimates are for a $95 \%$ confidence level. For each measured state point the combined uncertainty of the speed of sound is reported in Table 2 The combined uncertainties were rounded to the same number of digits as the speed-of-sound data.

\section{Discussion}

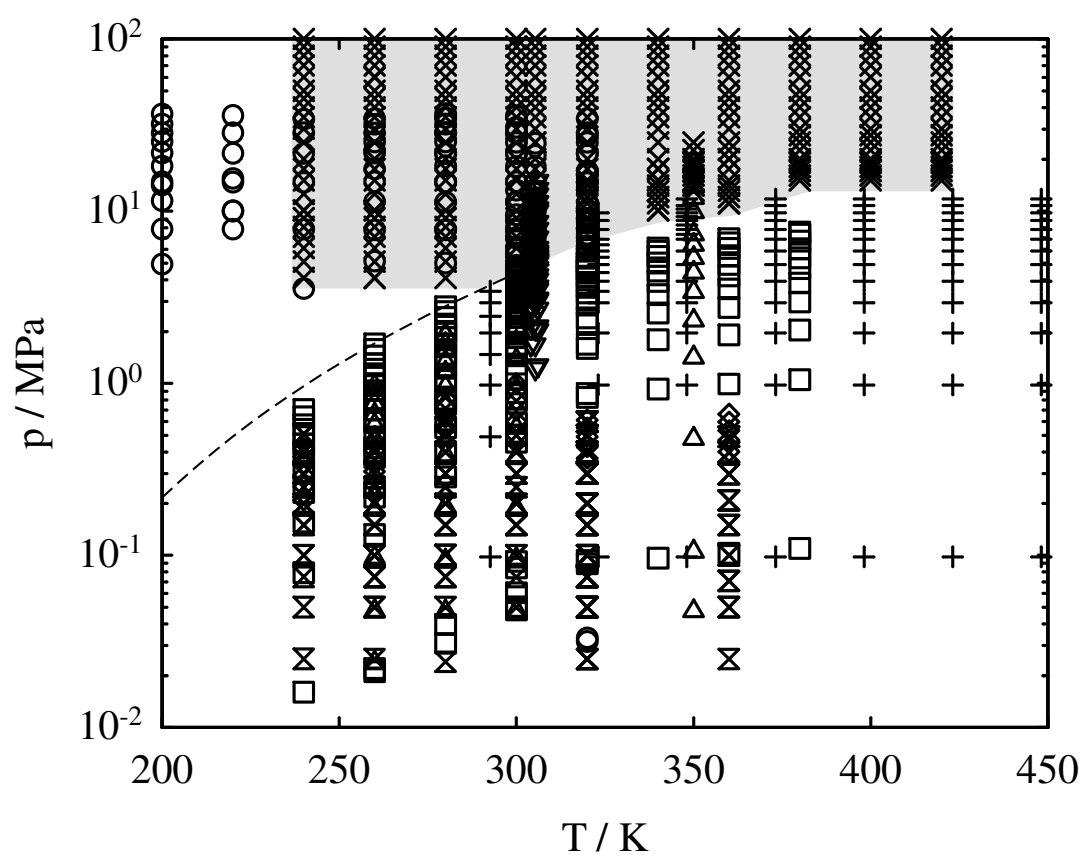

Fig. 3. Distribution of our measurements and literature data for the speed of sound in ethane in the $p, T$ plane. The grey area is the region of our measurements. Legend: $X$, This work; $\bigcirc$, Tsumura and Straty;

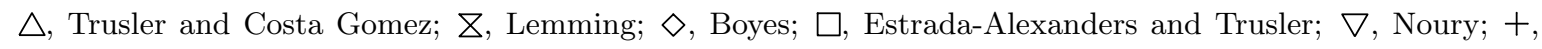
Terres et al.; $\diamond$, critical point and ----, vapor pressure calculated from the fundamental equation of state of Bücker and Wagner.

Experimental data for the speed of sound in ethane were published by several groups. Fig. 3 depicts the distribution of our data and literature data sets in the pressure-temperature plane. Lemming [10, Boyes [11, Colgate et al. [12], and Estrada-Alexanders and Trusler [9] measured speeds of sound in the gas region with a spherical resonator. The data sets of Trusler and Costa Gomez [3] and Terres et al. [13] also cover parts of the gas region and overlap with our data at low pressures at supercritical temperatures. Noury [14] measured the speed of sound in the critical region of ethane. Speeds of 
sound in saturated liquid ethane were measured by Poole and Aziz [15, Vangeel [16], and Tsumura and Straty [2]. Only the data set of Tsumura and Straty [2] covers a large part of the liquid region.

Fundamental equations of state for ethane in terms of the Helmholtz free energy were developed by Friend et al. [17, Span and Wagner [18, Bücker and Wagner [1, and Kunz and Wagner 19]. Younglove and Ely 20] published a thermal equation of state of the MBWR-type, which together with an equation for the isochoric ideal gas heat capacity can be represented as a fundamental equation of state. Since the Bücker and Wagner equation is the most accurate fundamental equation of state for ethane, it is chosen as the reference for the following discussion. In the remainder of this section, our data are compared with the equation of state of Bücker and Wagner [1, the data of Tsumura and Straty [2], and the data of Trusler and Costa Gomez [3].

In Figs. 4 to 6 , the relative deviations of our data, the data of Tsumura and Straty $[2]$ and Trusler and Costa Gomez [3] from the fundamental equation of state of Bücker and Wagner are plotted versus pressure for all measured isotherms. Since the deviations of speeds of sound calculated from the other fundamental equations of state and of the data of Terres et al. [13] and Noury [14 from the fundamental equation of state of Bücker and Wagner are in large parts much larger than the chosen scale of the figures, they have not been included in the deviation plots.

Our data agree with the fundamental equation of state within $0.1 \%$ with the exception of a few points at high pressures on the six isotherms up to $T=320 \mathrm{~K}$, where deviations up to $0.3 \%$ are observed. They generally show the same systematic deviation pattern as the data of Tsumura and Straty, and agree with them mostly within $0.03 \%$. Our data are very consistent, while the data of Tsumura and Straty scatter within their uncertainty of about $0.05 \%$. At T $=260 \mathrm{~K}$ our data are up to $0.04 \%$ higher than the Tsumura and Straty data, and at low pressures on the critical isotherm deviations up to $0.05 \%$ are found. Tsumura and Straty estimated the uncertainty of their data generally to $0.05 \%$. Only close to the critical point, that is at the lowest pressures at $T=300 \mathrm{~K}$ and on the critical isotherm $T=305.32 \mathrm{~K}$ the uncertainty is $0.1 \%$. Thus, the agreement between the two data sets is within the uncertainty the data of Tsumura and Straty.

At $T=350 \mathrm{~K}$, we compare our data with five spherical resonator data of Trusler and Costa Gomez in the pressure range between $10 \mathrm{MPa}$ and $20 \mathrm{MPa}$ where the two different measurement techniques overlap. The uncertainty of these data is $0.025 \%$. Again, both data sets show the same deviation pattern with a small maximum between $10 \mathrm{MPa}$ and $20 \mathrm{MPa}$. Our data are $0.03 \%$ higher than

the data of Trusler and Costa Gomez. Thus, the agreement is within their mutual uncertainties. The maximum of the deviations is also observed on all other supercritical isotherms, being shifted to higher pressures with increasing temperature. This systematic deviation pattern may result from certain terms in the fundamental equation of state. This conclusion is warranted because of the low uncertainty of our experimental data. Conversely, the present experimental data should result in future formulations of the Helmholtz energy of ethane with different terms.

\section{Conclusions}

We have measured the speed of sound in liquid and supercritical ethane between $240 \mathrm{~K}$ and $420 \mathrm{~K}$ up to $100 \mathrm{MPa}$ with high accuracy. This range exceeds the previously available speed of sound data for ethane considerably as does the accuracy. Therefore, this data set provides significant leverage to 
improve the formulation of the Helmholtz energy for ethane.

\section{Acknowledgements}

This work was motivated by Dr. Eric Lemmon, National Institute of Standards and Technology in Boulder. All equation of state calculations in this work were carried out with the NIST reference data base REFPROP 9.1 [21]. We thank Christian Rosek for his assistance during parts of the measurement campaign.

\section{List of Symbols}

$\begin{array}{ll}c & \text { speed of sound }\left(\mathrm{m} \cdot \mathrm{s}^{-1}\right) \\ l & \text { distance between quartz crystal and reflector }(\mathrm{m}) \\ \Delta l & \text { acoustic path length }(\mathrm{m}) \\ p & \text { pressure }(\mathrm{MPa}) \\ T & \text { temperature }(\mathrm{K}) \\ U_{\mathrm{c}} & \text { combined uncertainty (at the } 95 \% \text { confidence level) } \\ u & \text { expanded uncertainty (at the } 95 \% \text { confidence level) }\end{array}$



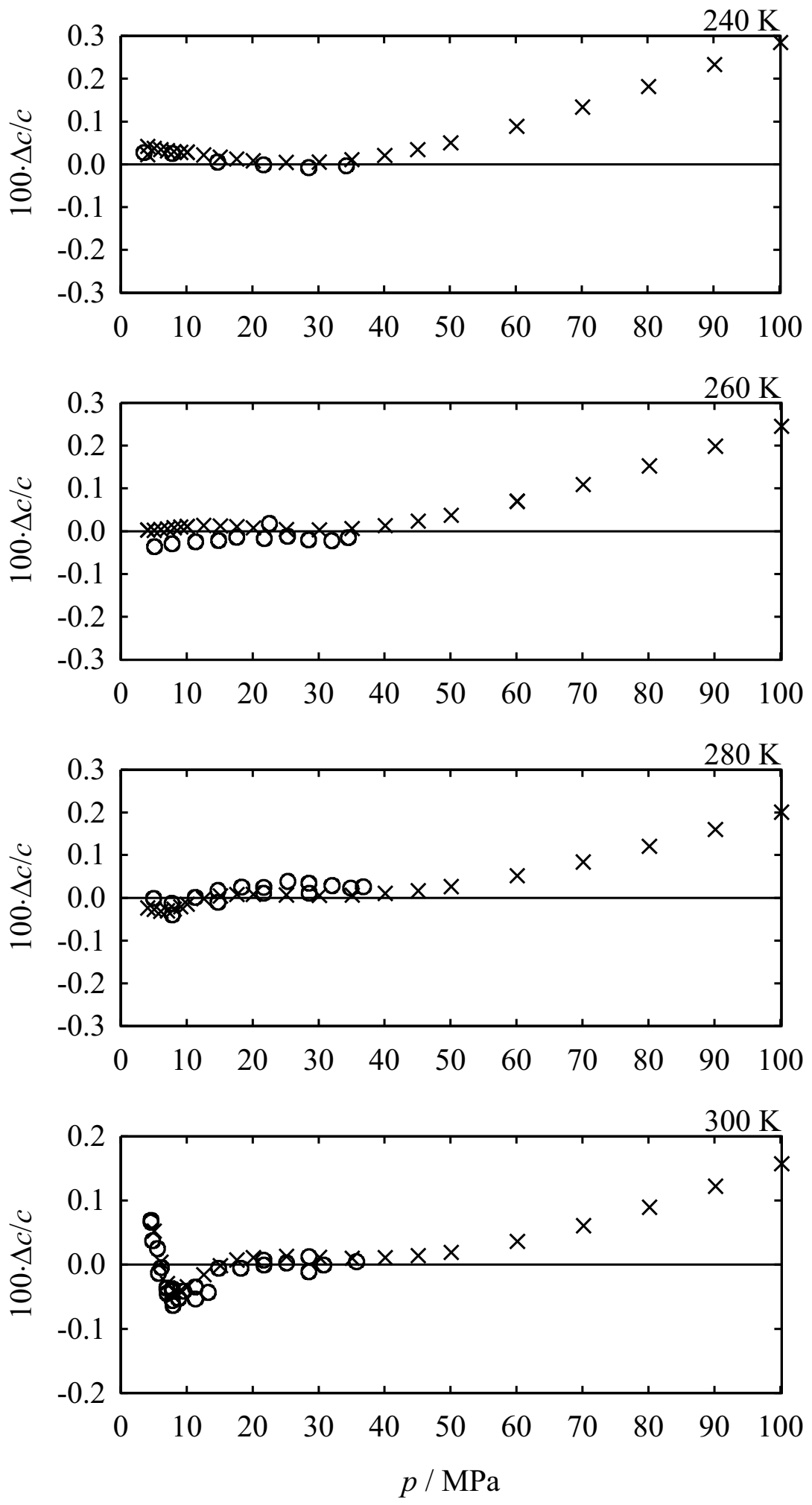

Fig. 4. Relative deviations $\Delta c=c$ (expt.) $-c$ (calc.) of experimental speeds of sound $c$ (expt.) in ethane and literature data from values $c$ (calc.) calculated from the fundamental equation of state of Bücker and Wagner as a function of pressure at $240 \mathrm{~K}, 260 \mathrm{~K}, 280 \mathrm{~K}$ and $300 \mathrm{~K}$. Legend: X, This work; O, Tsumura and Straty. 

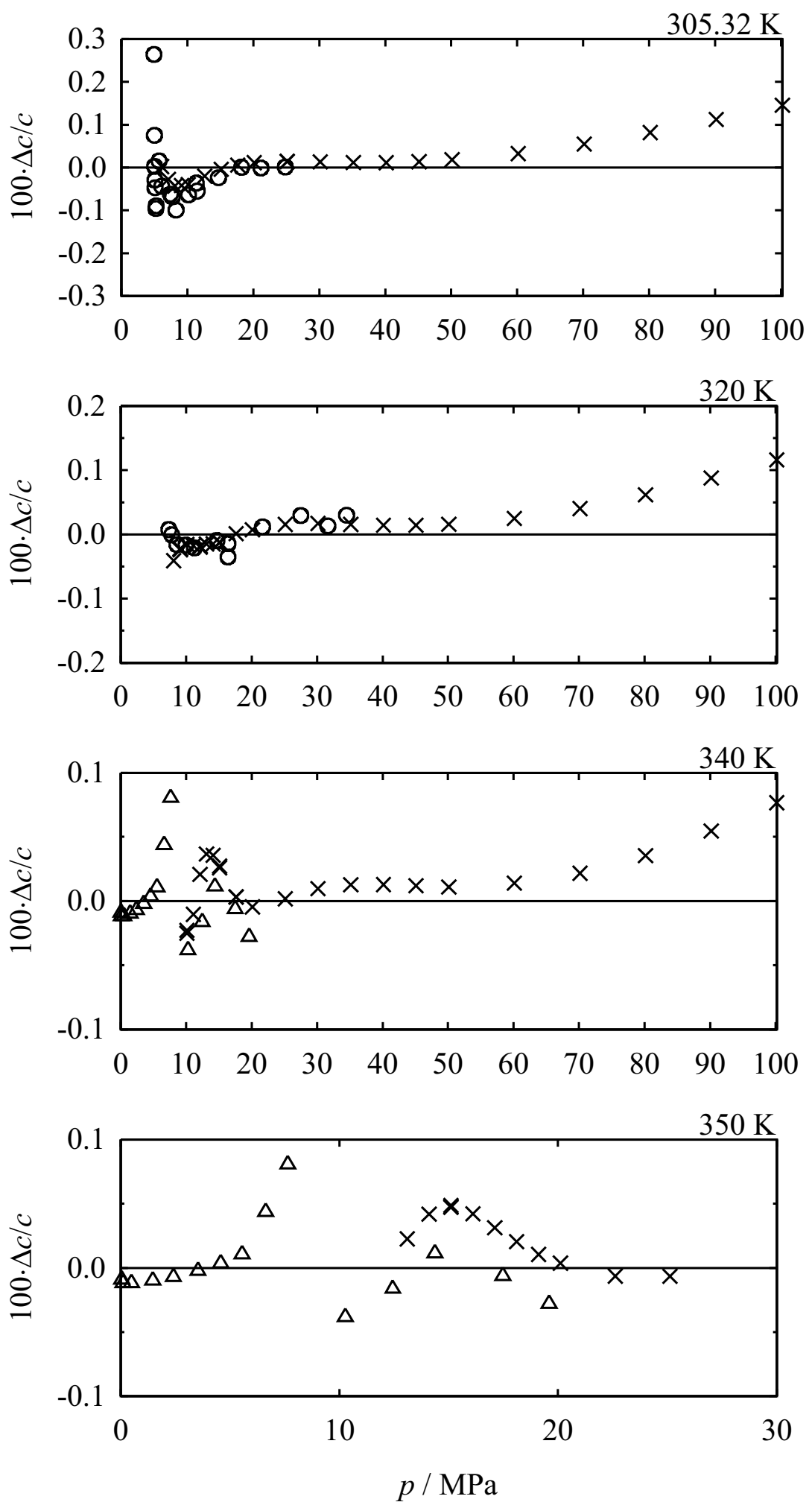

Fig. 5. Relative deviations $\Delta c=c$ (expt.) $-c$ (calc.) of experimental speeds of sound $c$ (expt.) in ethane and literature data from values $c$ (calc.) calculated from the fundamental equation of state of Bücker and Wagner as a function of pressure at $305.32 \mathrm{~K}, 320 \mathrm{~K}, 340 \mathrm{~K}$ and $360 \mathrm{~K}$. Legend: X, This work; O, Tsumura and Straty; $\triangle$, Trusler and Costa Gomez at $350 \mathrm{~K}$. 

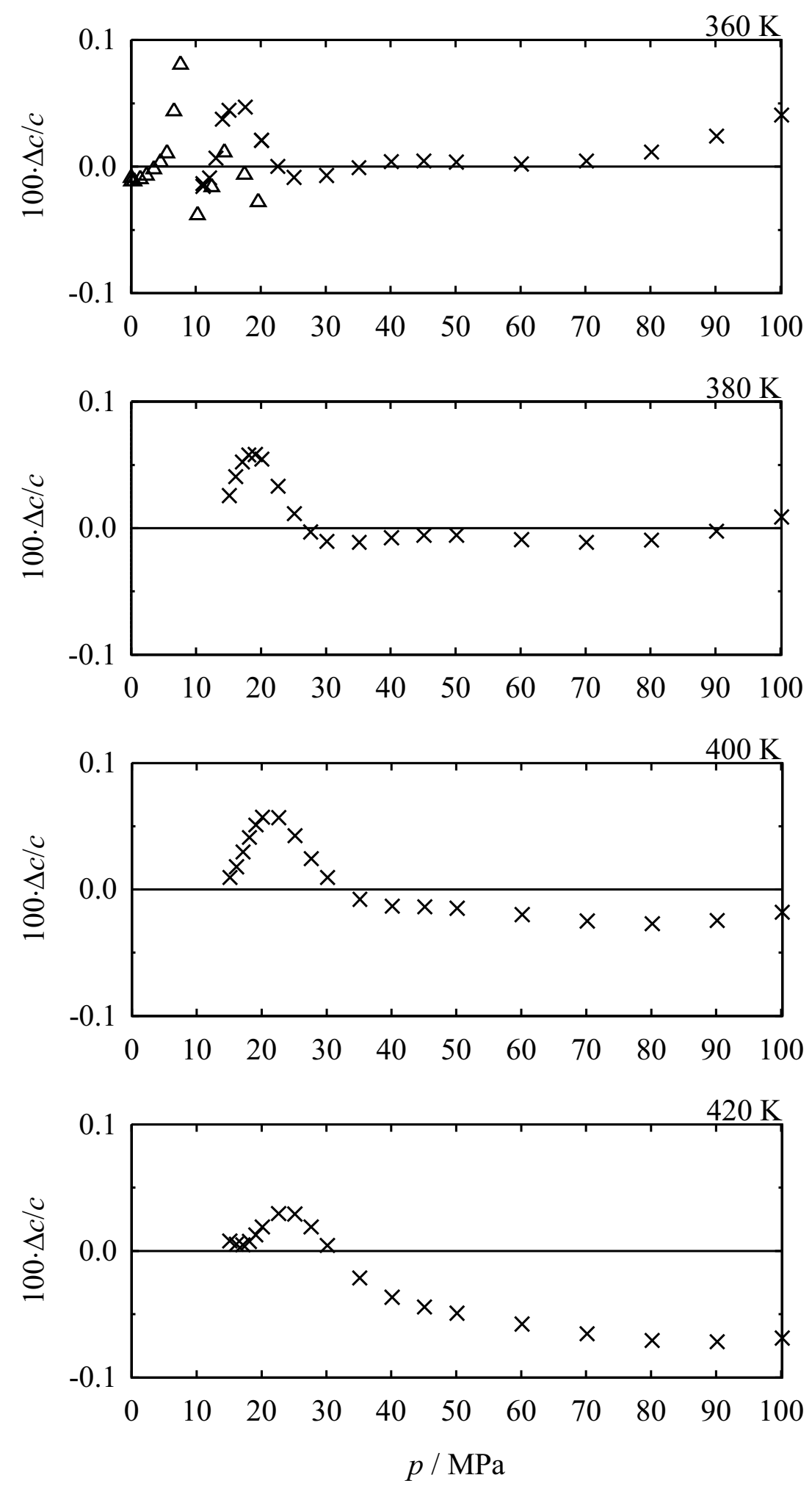

Fig. 6. Relative deviations $\Delta c=c$ (expt.) $-c$ (calc.) of experimental speeds of sound $c$ (expt.) in ethane and literature data from values $c$ (calc.) calculated from the fundamental equation of state of Bücker and Wagner as a function of pressure at $360 \mathrm{~K}, 380 \mathrm{~K}, 400 \mathrm{~K}$ and $420 \mathrm{~K}$. Legend: $\times$, This work; $\triangle$, Trusler and Costa Gomez at $350 \mathrm{~K}$. 


\section{References}

[1] D. Bücker, W. Wagner, J. Phys. Chem. Ref. Data 35 (2006) 205-266. doi:10.1063/1.1859286.

[2] R. Tsumura, G. C. Straty, J. Chem. Thermodyn. 4 (1977) 195-200. doi:10.1016/0011-2275(77) 90165-5.

[3] J. P. M. Trusler, M. F. Costa Gomez, Research report for the GERG project "Fundamental equations for calorific properties", London, 1996.

[4] K. Meier, The pulse-echo method for high-precision measurements of the speed of sound in fluids, Habilitation Thesis, Faculty of Mechanical Engineering, Helmut-Schmidt-University, Hamburg, 2006.

[5] K. Meier, S. Kabelac, Rev. Sci. Instrum. 77 (2006) 123903. doi:10.1063/1.2400019.

[6] K. Meier, S. Kabelac, J. Chem. Eng. Data 57 (2012) 3391-3398. doi 10.1021/je300466a.

[7] K. Meier, S. Kabelac, J. Chem. Eng. Data 58 (2013) 1621-1628. doi 10.1021/je301344y.

[8] M. J. P. Muringer, N. J. Trappeniers, S. N. Biswas, Phys. Chem. Liq. 14 (1985) 273-296. doi 10. 1080/00319108508080991.

[9] A. F. Estrada-Alexanders, J. P. M. Trusler, J. Chem. Thermodyn. 29 (1997) 991-1015. doi 10. $1006 /$ jcht.1997.0217.

[10] W. Lemming, Experimentelle Bestimmung akustischer und thermischer Virialkoeffizienten von Arbeitsstoffen der Energietechnik, Fortschr.-Ber. VDI Reihe 19 Nr. 32, 1989.

[11] S. J. Boyes, The speed of sound in gases with application to equations of state and sonic nozzles, Ph.D. Thesis, University College London, 1992.

[12] S. O. Colgate, A. Sivaraman, C. Dejsupa, Fluid Phase Equilbr. 76 (1992) 175-185. doi 10.1016/ 0378-3812(92)85086-N.

[13] E. Terres, W. Jahn, H. Reissmann, Brennst.-Chem. 38 (1957) 129-160.

[14] J. Noury, Comptes Rendus 234 (1952) 303-305.

[15] G. R. Poole, R. A. Aziz, Can. J. Phys. 50 (1972) 721-727. doi:10.1139/p72-102.

[16] E. Vangeel, private communication with NBS, Boulder. Data published in [22], 1976.

[17] D. G. Friend, H. Ingham, J. F. Ely, J. Phys. Chem. Ref. Data 20 (1991) 275-347. doi 10.1063/ 1.555881

[18] R. Span, W. Wagner, Int. J. Thermophys. 24 (2003) 41-109. doi:10.1023/A:1022310214958 
[19] O. Kunz, R. Klimeck, W. Wagner, M. Jaeschke, The GERG-2004 wide-range equation of state for natural gases and other mixtures, GERG Technical Monograph 15, VDI Verlag, Düsseldorf, 2007.

[20] B. A. Younglove, J. F. Ely, J. Phys. Chem. Ref. Data 16 (1987) 577-798. doi 10.1063/1.555785.

[21] E. W. Lemmon, M. L. Huber, M. O. McLinden, Refprop 9.1, NIST Standard Reference Database 21, NIST, Boulder, 2013.

[22] R. D. Goodwin, H. M. Roder, G. C. Straty, Thermophysical properties of ethane from 90 to 600 K at pressures to 700 bar, NBS Technical Note 684, National Bureau of Standards, Boulder, 1976. 